\title{
Nonregular Boundary Value Problem for the Cauchy-Riemann Operator
}

\author{
Ibrahim Ly, Sadou Tao \\ Département de Mathématiques, Université Joseph KI-ZERBO, Ouagadougou, Burkina Faso \\ Email: lyibrahim@gmx.de, sadoutao@yahoo.fr
}

How to cite this paper: Ly, I. and Tao, S. (2020) Nonregular Boundary Value Problem for the Cauchy-Riemann Operator. Advances in Pure Mathematics, 10, 623-630. https://doi.org/10.4236/apm.2020.1011038

Received: September 1, 2020

Accepted: November 9, 2020

Published: November 12, 2020

Copyright $\odot 2020$ by author(s) and Scientific Research Publishing Inc. This work is licensed under the Creative Commons Attribution International License (CC BY 4.0).

http://creativecommons.org/licenses/by/4.0/

\begin{abstract}
The purpose of the research is to assign a formally exact elliptic complex of length two to the Cauchy-Riemann Operator. The Neumann problem for this complex in a bounded domain with smooth boundary in $\mathbb{R}^{2}$ will be studied, helping therefore to solve a usual boundary value problem for the Cauchy-Riemann operator.
\end{abstract}

\section{Keywords}

Cauchy-Riemann Operator, Boundary Value Problem, Normal Solvability

\section{Introduction}

Most first order linear differential operators of geometric origin are Dirac operators. Dirac operators on Riemannian manifolds are of fundamental importance in differential geometry. A $(\ell \times k)$-matrix $D$ of first order scalar differential operators with constant coefficients in $\mathbb{R}^{n}$ is said to be a Dirac type operator, if $D^{*} D=-E_{k} \Delta$, where $E_{k}$ is the identity $(k \times k)$-matrix, $\Delta$ the (non-positive) Laplace operator in $\mathbb{R}^{n}$, and $D^{*}$ is the formal adjoint of $D$. As usual, we denote by $\sigma^{1}(D)(\xi)$ the principal symbol of $D$. The rank of this matrix is equal to $k$ for all $\xi \in \mathbb{R}^{n} \backslash\{0\}$. It follows that every Dirac type operator is overdetermined elliptic. For $\ell=k$, such operators are called elliptic in the classical literature.

In this paper, we firstly restrict our discussion to a boundary value problem related to the Cauchy-Riemann operator, which is a Dirac type operator. A similar work has been done by [1] for the Fueter-operator, but using a cohomology-method. When studying a boundary value problem, we usually look for conditions which guarantee that the solution exists, is unique and depends continuously on the problem data. Let $\mathcal{X} \Subset \mathbb{R}^{2}$ be a domain with smooth boun- 
dary. Given functions $f: \mathcal{X} \rightarrow \mathbb{R}^{2}$ and $u_{1,0}: \partial \mathcal{X} \rightarrow \mathbb{R}^{2}$, find a solution $u$ to $D u=f(x, u)$ in $\mathcal{X}$, whose first component coincides with $u_{1,0}$ on the boundary of $\mathcal{X}$, and where $D$ is the Cauchy-Riemann operator.

It is important to point out that no attempt has been made here to develop any general theory. The Atiyah-Patodi-Singer index theorem drew mathematicians' attention to the so-called spectral boundary conditions for Dirac operators, thus highlighting an idea of Calderón (1963). For an excellent exposition of spectral elliptic boundary problems for Dirac operators, we refer to [2].

The scheme of the article can be declined in the following way: In Section 2, we show that boundary value problem related to the Cauchy-Riemann operator in the plane satisfies the Lopatinskii condition. The paragraph Section 3 is devoted to proving a necessary condition to the existence of a solution to our problem being our main result. To this end, finding a compatible complex to $D u=f(x, u)$ in $\mathcal{X}$ and $u_{1}=u_{1,0}$ on the boundary of $\mathcal{X}$ will be highlighted in Section 4. Before coming to some generalisations in Section 6, the corresponding Hodge theory to our problem will be handled in Section 5.

\section{A Classical Problem}

Suppose $\mathcal{X}$ is a bounded domain with smooth boundary in the complex plane $\mathbb{C}$. Identifying $\mathbb{C}$ with $\mathbb{R}^{2}$ under the complex structure $z=x_{1}+i x_{2}$, we consider the inhomogeneous system

$$
\begin{aligned}
& \partial_{1} u_{1}-\partial_{2} u_{2}=f_{1}, \\
& \partial_{2} u_{1}+\partial_{1} u_{2}=f_{2}
\end{aligned}
$$

for an unknown function $u=u_{1}+i u_{2}$ in $\mathcal{X}$, satisfying the boundary condition

$$
u_{1} \Gamma_{\partial \mathcal{X}}=u_{1,0} \text {, }
$$

where $f=f_{1}+i f_{2}$ and $u_{1,0}$ are prescribed functions in $\mathcal{X}$ and on $\partial \mathcal{X}$, respectively. Note that (2.1) just amounts to the inhomogeneous Cauchy-Riemann system in the plane.

When assuming $f \in H^{s-1}(\mathcal{X})$ and $u_{1,0} \in H^{s-1 / 2}(\partial \mathcal{X})$ and looking for a solution $u \in H^{s}(\mathcal{X})$, one easily verifies that this boundary value problem is Fredholm for each $s \in \mathbb{N}$. Since the Fredholm property is actually equivalent to the ellipticity, we may deduce that the problem (2.1), (2.2) fulfills the Lopatinskii condition. However, these arguments are opposite to what the Lopatinskii condition is aimed at. We present a direct proof.

Theorem 2.1. The boundary value problem (2.1), (2.2) satisfies the Lopatinskii condition.

Proof. The Lopatinskii condition is local, and so it suffices to verify it in a small neighbourhood of any point $x_{0} \in \partial \mathcal{X}$. Since the boundary of $\mathcal{X}$ is smooth, there is a conformal mapping of $B\left(x_{0}, \varepsilon\right) \cap \overline{\mathcal{X}}$, with $\varepsilon>0$ small enough, to the upper half-plane $\left\{x \in \mathbb{R}^{2}: x_{2} \geq 0\right\}$, such that the curve $B\left(x_{0}, \varepsilon\right) \cap \partial \mathcal{X}$ is mapped into the $x_{1}$-axis, which is due to the Riemann theorem. Moreover, the Cauchy-Riemann system survives under conformal map- 
pings. Hence, we can assume without restriction of generality that $\mathcal{X}$ is the upper half-plane. For each fixed $x_{2} \geq 0$, we apply the Fourier transformation in $x_{1}$ to both Equation (2.1) and boundary condition (2.2). This gives

$$
\begin{aligned}
& \left(i \xi_{1}\right) \hat{u}_{1}\left(\xi_{1}, x_{2}\right)-\partial_{2} \hat{u}_{2}\left(\xi_{1}, x_{2}\right)=\hat{f}_{1}\left(\xi_{1}, x_{2}\right), \\
& \partial_{2} \hat{u}_{1}\left(\xi_{1}, x_{2}\right)+\left(i \xi_{1}\right) \hat{u}_{2}\left(\xi_{1}, x_{2}\right)=\hat{f}_{2}\left(\xi_{1}, x_{2}\right)
\end{aligned}
$$

for all $x_{2}>0$ as well as an initial condition $\hat{u}_{1}\left(\xi_{1}, 0\right)=\hat{u}_{1,0}\left(\xi_{1}\right)$, the "hat" meaning Fourier transformation in $x_{1}$. From this we read off the boundary symbol of our problem, namely

$$
\sigma_{\partial}(\cdot)\left(x_{1}, \xi_{1}\right): \mathcal{S}\left(\mathbb{R}_{\geq 0}, \mathbb{C}^{2}\right) \rightarrow \stackrel{\mathcal{S}\left(\mathbb{R}_{\geq 0}, \mathbb{C}^{2}\right)}{\oplus}
$$

where $\mathcal{S}\left(\mathbb{R}_{\geq 0}, \mathbb{C}^{2}\right)$ is the space of all rapidly decreasing functions on the half-axis $\left\{x_{2} \in \mathbb{R}: x_{2} \geq 0\right\}$ with values in $\mathbb{C}^{2}$, and

$$
\sigma_{\partial}(\cdot)\left(x_{1}, \xi_{1}\right) v=\left(\begin{array}{c}
\partial_{2} v-A v \\
v_{1}(0)
\end{array}\right)
$$

with $A=\left(\begin{array}{cc}0 & -i \xi_{1} \\ i \xi_{1} & 0\end{array}\right)$.

The Lopatinskii condition just amounts to saying that (2.4) is a bijective mapping for all $\xi_{1} \in \mathbb{R} \backslash\{0\}$. There is no loss of generality in assuming that $\xi_{1}>0$. The general solution of the homogeneous system $\partial_{2} v-A v=0$ for $x_{2}>0$ with initial condition $v_{1}(0)=v_{1,0}$ has the form

$$
\begin{aligned}
& v_{1}\left(x_{2}\right)=v_{1,0} \cosh \left(\xi_{1} x_{2}\right)-i c_{2} \sinh \left(\xi_{1} x_{2}\right), \\
& v_{2}\left(x_{2}\right)=i v_{1,0} \sinh \left(\xi_{1} x_{2}\right)+c_{2} \cosh \left(\xi_{1} x_{2}\right),
\end{aligned}
$$

$c_{2}$ being an arbitrary constant. If we require a solution in $\mathcal{S}\left(\mathbb{R}_{\geq 0}, \mathbb{C}^{2}\right)$, we have the only choice for the constant $c_{2}$, namely $c_{2}=-i v_{1,0}$. This proves the injectivity of (2.4).

To show that (2.4) is surjective for $\xi_{1}>0$, we fix $g \in \mathcal{S}\left(\mathbb{R}_{\geq 0}, \mathbb{C}^{2}\right)$. An easy computation shows that

$$
\begin{aligned}
v\left(x_{2}\right)= & v_{1,0} \exp \left(-\xi_{1} x_{2}\right)\left(\begin{array}{c}
1 \\
-i
\end{array}\right) \\
& +\int_{0}^{x_{2}}\left(\begin{array}{cc}
\cosh \xi_{1}\left(x_{2}-\vartheta\right) & -i \sinh \xi_{1}\left(x_{2}-\vartheta\right) \\
i \sinh \xi_{1}\left(x_{2}-\vartheta\right) & \cosh \xi_{1}\left(x_{2}-\vartheta\right)
\end{array}\right) g(\vartheta) \mathrm{d} \vartheta \\
& +c_{2}\left(\begin{array}{c}
-i \sinh \left(\xi_{1} x_{2}\right) \\
\cosh \left(\xi_{1} x_{2}\right)
\end{array}\right)
\end{aligned}
$$

is a general solution to the system $\partial_{2} v-A v=g$ for $x_{2}>0$ with initial data $v_{1}(0)=v_{1,0}$. This solution is parametrised by a constant $c_{2}$ and it fails to belong to $\mathcal{S}\left(\mathbb{R}_{\geq 0}, \mathbb{C}^{2}\right)$ for an arbitrary choice of $c_{2}$. However, there is a unique constant $c_{2}$ for which it is the case. Indeed, the sum of the last two terms on the right-hand side of (2.7) is 


$$
\begin{aligned}
& \frac{1}{2}\left(\int_{0}^{x_{2}} \exp \xi_{1}\left(x_{2}-\vartheta\right)\left(i g_{1}(\vartheta)+g_{2}(\vartheta)\right) \mathrm{d} \vartheta+c_{2} \exp \left(\xi_{1} x_{2}\right)\right)\left(\begin{array}{c}
-i \\
1
\end{array}\right) \\
& +\frac{1}{2}\left(\int_{0}^{x_{2}} \exp \left(-\xi_{1}\left(x_{2}-\vartheta\right)\right)\left(-i g_{1}(\vartheta)+g_{2}(\vartheta)\right) \mathrm{d} \vartheta+c_{2} \exp \left(-\xi_{1} x_{2}\right)\right)\left(\begin{array}{l}
i \\
1
\end{array}\right),
\end{aligned}
$$

as is easy to verify. Choose $c_{2}$ in such a way that the first term would vanish at infinity, i.e.

$$
c_{2}=-\int_{0}^{\infty} \exp \left(-\xi_{1} \vartheta\right)\left(i g_{1}(\vartheta)+g_{2}(\vartheta)\right) \mathrm{d} \vartheta .
$$

Then it becomes

$$
-\frac{1}{2} \int_{x_{2}}^{\infty} \exp \xi_{1}\left(x_{2}-\vartheta\right)\left(i g_{1}(\vartheta)+g_{2}(\vartheta)\right) \mathrm{d} \vartheta\left(\begin{array}{c}
-i \\
1
\end{array}\right),
$$

which is a rapidly decreasing function of $x_{2} \in \mathbb{R}_{\geq 0}$. Since the second term is rapidly decreasing, the surjectivity follows.

The proof of Theorem 2.1 shows that the verification of the Lopatinskii condition is actually as hard as the construction of a parametrix to the boundary value problem.

\section{Existence of Solution}

Let $D$ be the Cauchy-Riemann operator with constant coefficients in $\mathbb{R}^{2}$, thus satisfying $D^{*} D=-E_{2} \Delta$.

Suppose $\mathcal{X}$ is a bounded domain with smooth boundary in $\mathbb{R}^{2}$ and $f$ a given function on $\mathcal{X}$ with values in $\mathbb{R}^{2}$ of Sobolev class $H^{s-1}\left(\mathcal{X}, \mathbb{R}^{2}\right), s$ being a natural number. We will write $H^{s}(\mathcal{X})$ for $H^{s}\left(\mathcal{X}, \mathbb{R}^{2}\right)$ if no confusion can arise. Consider the inhomogeneous nonlinear Dirac type equation $D u=f(x, u)$ for an unknown function $u \in H^{s}\left(\mathcal{X}, \mathbb{R}^{2}\right)$.

The operator $D$ is elliptic, and so all generalised solutions of $D u=f(x, u)$ are in fact locally in the space $H^{s}\left(\mathcal{X}, \mathbb{R}^{2}\right)$. We interpret a solution $u$ as a column of Sobolev functions on $\mathcal{X}$, i.e.

$$
u=\left(\begin{array}{l}
u_{1} \\
u_{2}
\end{array}\right)
$$

where $u_{1}$ is a function on $\mathcal{X}$ with real values and $u_{2}$ takes its values in $\mathbb{R}$.

The determination of a solution $u$ of $D u=f(x, u)$ by means of its "scalar" component $u_{1} \in H^{s}(\mathcal{X})$ is a problem going back to the classical result of the reconstruction of a holomorphic function from its real part. It is studied in [3], cf. Section 1.2.5. We strengthen this problem in the following way. Let $u_{1,0} \in H^{s-1 / 2}(\partial \mathcal{X})$ be a prescribed function on the boundary of $\mathcal{X}$. Find a solution $u$ to $D u=f(x, u)$ in $\mathcal{X}$, such that $u_{1}=u_{1,0}$ on $\partial \mathcal{X}$.

We first find a necessary condition for the solvability of this problem. For convenience of reference we designate it as

$$
\begin{aligned}
& D u=f(x, u) \text { in } \mathcal{X}, \\
& u_{1}=u_{1,0} \text { on } \partial \mathcal{X},
\end{aligned}
$$


cf. (2.1), (2.2). Note that in one dimension (3.1) is precisely the Cauchy problem for the Dirac type equation $D u=f(x, u)$.

Write $D=\left(D_{1}, D_{2}\right)$, where $D_{1}$ is the first column of the matrix $D$ and $D_{2}$ the complementary-matrix of $D$. Since

$$
D^{*} D=\left(\begin{array}{l}
D_{1}^{*} \\
D_{2}^{*}
\end{array}\right)\left(D_{1}, D_{2}\right)=\left(\begin{array}{ll}
D_{1}^{*} D_{1} & D_{1}^{*} D_{2} \\
D_{2}^{*} D_{1} & D_{2}^{*} D_{2}
\end{array}\right)=-E_{2} \Delta,
$$

it follows that

$$
\begin{aligned}
& D_{1}^{*} D_{1}=-\Delta, \quad D_{1}^{*} D_{2}=0, \\
& D_{2}^{*} D_{1}=0, \quad D_{2}^{*} D_{2}=-\Delta .
\end{aligned}
$$

The first and the last equalities of (3.2) imply that both $D_{1}$ and $D_{2}$ are Dirac type operators.

Lemma 3.1. For a function $u \in H^{s}(\mathcal{X})$ to be a solution of (3.1) it is necessary that $u_{1}$ satisfy

$$
\begin{aligned}
& -\Delta u_{1}=D_{1}^{*} f(x, u) \text { in } \mathcal{X}, \\
& u_{1}=u_{1,0} \text { on } \partial \mathcal{X} .
\end{aligned}
$$

Proof. The equality $D u=f(x, u)$ is obviously equivalent to the equality $D_{1} u_{1}+D_{2} u_{2}=f(x, u)$ in $\mathcal{X}$. Applying the operator $D_{1}^{*}$ to both sides of the latter equality, we obtain, by (3.2),

$$
D_{1}^{*} D_{1} u_{1}=D_{1}^{*} f(x, u)-D_{1}^{*} D_{2} u_{2}=D_{1}^{*} f(x, u),
$$

and this is precisely the assertion of the lemma.

Hence, $u_{1}$ should be a solution of the Dirichlet problem (3.3) in $\mathcal{X}$ with given data $D_{1}^{*} f$ and $u_{1,0}$. Since the Dirichlet problem is uniquely solvable, we will assume from now on that the function $u_{1} \in H^{s}(\mathcal{X})$ is determined from (3.3). We are thus left with the task of finding the remaining components $u_{2}$ of $u$, namely to solving $D_{2} u_{2}=f(x, u)-D_{1} u_{1}$ in the domain of $\mathcal{X}$.

To this end, we consider in Section 4 the elliptic complex related to our Cauchy-Riemann operator

\section{A Compatibility Complex}

Let us state our lemma.

Lemma 4.1. The differential operators $D_{2}^{*}$ and $D_{1}$ fit together to form an elliptic complex over $\mathcal{X}$

$$
0 \rightarrow C^{\infty}(\overline{\mathcal{X}}) \stackrel{D_{2}}{\longrightarrow} C^{\infty}(\overline{\mathcal{X}}) \stackrel{D_{1}^{*}}{\longrightarrow} C^{\infty}(\overline{\mathcal{X}}) \rightarrow 0 .
$$

Proof. The Laplacian $\Delta^{0}$ of (4.1) at step 0 is elliptic, for

$$
\Delta^{0}=D_{2}^{*} D_{2}=-E \Delta
$$

according to (3.2).

Since $D$ is a square matrix of scalar differential operators with constant coefficients, we deduce that 


$$
-E \Delta=D D^{*}=\left(D_{1}, D_{2}\right)\left(\begin{array}{l}
D_{1}^{*} \\
D_{2}^{*}
\end{array}\right)=D_{1} D_{1}^{*}+D_{2} D_{2}^{*} .
$$

Hence it follows that the Laplacian $\Delta^{1}=D_{1} D_{1}^{*}+D_{2} D_{2}^{*}$ of complex (4.1) at step 1 is elliptic.

Finally, the Laplacian $\Delta^{2}$ of (4.1) at step 2 is elliptic, for

$$
\Delta^{2}=D_{1}^{*} D_{1}=-\Delta
$$

by (3.2).

\section{Hodge Theory}

The Hodge theory is a very important technical tool for solving partial differential equations, in particular for solving Neumann problems.

In this section, we define two very important spaces before considering a "weak version" of the Neumann problem for our elliptic complex (6.2), namely

$$
N^{1}(\mathcal{X})=\left\{u \in \mathbb{C}^{\infty}(\overline{\mathcal{X}}): n(u)=n\left(D_{1}^{*} u\right)=0 \text { on } \partial \mathcal{X}\right\}
$$

and

$$
H^{1}(\mathcal{X})=\left\{u \in \mathbb{C}^{\infty}(\overline{\mathcal{X}}): n(u)=0 \text { on } \partial \mathcal{X} \text { and } D_{1}^{*} u=D_{2}^{*} u=0 \text { in } \mathcal{X}\right\}
$$

The spaces $N^{1}(\mathcal{X})$ and $H^{1}(\mathcal{X})$ are called Neumann and Harmonic spaces, respectively.

By $n(u)$ and $n\left(D_{1}^{*}\right)$ are meant the Cauchy data of $u$ on $\partial \mathcal{X}$, with respect to the differential operators $D_{2}^{*}$, and $D_{1}$, respectively, cf. Section 3.2.2 in [3].

Moreover, we precise that for all $u \in C^{\infty}(\overline{\mathcal{X}}), n(u)=0 \Leftrightarrow$ $\left(\sigma^{1}\left(D_{2}\right)(v)\right)^{*} u=0$ on $\partial \mathcal{X}$, and $n\left(D_{1}^{*} u\right)=0 \Leftrightarrow\left(\sigma^{1}\left(D_{1}\right)(v)\right) u=0$ on $\partial \mathcal{X}$, where $v(x)$ is the outward normal vector of the boundary of $\mathcal{X}$ at a point $x \in \partial \mathcal{X}$.

The Neumann problem for complex (6.2) on the manifold $\overline{\mathcal{X}}$ in the $C^{\infty}$ setting consists in the following:

(NP): Let be $f(x, u)$ in $C^{\infty}(\mathcal{X})$. Find a $u \in N^{1}(\mathcal{X})$ to $\Delta^{1} u=f(x, u)$

(Solvability): We say that the Neumann problem related to our complex is solvable at step-1, if:

(1) $H^{1}(\mathcal{X})$ is of finite dimension

(2) The equation $\Delta^{1} u=f(x, u)$ has a solution $u \in N^{1}(\mathcal{X})$ for each $f(x, u) \in C^{\infty}(\overline{\mathcal{X}})$ with $f \perp H^{1}(\mathcal{X})$

It is a well known result that Neumann problems are solvable for certain classes of manifolds $\mathcal{X}$, namely for manifolds which are strictly pseudoconvex with respect to the considered complex.

We now state the Hodge theory theorem related to our complex

Theorem 5.1. Let $\mathcal{X}$ be a strict pseudoconvex domain. There exist continuous operator $H: C^{\infty}(\overline{\mathcal{X}}) \rightarrow H^{1}(\mathcal{X})$ and $N: C^{\infty}(\overline{\mathcal{X}}) \rightarrow N^{1}(\mathcal{X})$ such that

(1) $f=H f+D_{2} D_{2}^{*} N f+D_{1} D_{1}^{*} N f$ for each $f \in C^{\infty}(\overline{\mathcal{X}})$

(2) If $f \in C^{\infty}(\overline{\mathcal{X}})$ and $D_{1}^{*} f=0$, then $D_{1}^{*} N f=0$ 
Proof. cf. [4]

In Section 3, we were left with the task of finding the remaining components $u_{2}$ of $u$, namely to solving

$$
D_{2} u_{2}=f(x, u)-D_{1} u_{1}
$$

in the domain of $\mathcal{X}$.

We now derive a sufficient condition for the solvability of (5.1)

Theorem 5.2. For Equation (5.1) to be solvable, it is sufficient that

$$
(f, h)-\int_{\partial \mathcal{X}}\left(t\left(u_{1}\right), n(h)\right) \mathrm{d} s=0
$$

for each $h \in H^{1}(\mathcal{X})$, where $t(u)$ and $n(h)$ are called the Dirichlet and Neumann data, respectively, cf. [5], and $\mathrm{d} s$ is the surface measure.

Proof. Using the Green formula cf. Section 3.2.2 in [5], we obtain that for $h \in H^{1}(\mathcal{X})$

$$
(f, h)-\int_{\partial \mathcal{X}}\left(t\left(u_{1}\right), n(h)\right) \mathrm{d} s=0 \Leftrightarrow(f, h)-\left(u_{1}, D_{1}^{*} h\right)-\int_{\partial \mathcal{X}}\left(t\left(u_{1}\right), n(h)\right) \mathrm{d} s=0
$$

Setting $F=f(x, u)-D_{1} u_{1}$, and from the Hodge theory, we readily get that $H F=0$ allowing to choose $u_{2}$ as $u_{2}=D_{2}^{*} N F$.

\section{Some Generalisations}

Let $D$ be a general Dirac operator in $\mathbb{R}^{n}$ given by a $(k \times k)$-matrix. Write

$$
D=(A, C)
$$

where $A$ is the first column of the matrix $D$ and $C$ the complementary $(k \times(k-1))$-matrix of scalar differential operators.

Since

$$
D^{*} D=\left(\begin{array}{l}
A^{*} \\
C^{*}
\end{array}\right)(A, C)=\left(\begin{array}{ll}
A^{*} A & A^{*} C \\
C^{*} A & C^{*} C
\end{array}\right)=-E_{k} \Delta,
$$

it follows that

$$
\begin{aligned}
& A^{*} A=-\Delta, \quad A^{*} C=0, \\
& C^{*} A=0, \quad C^{*} C=-E_{k-1} \Delta .
\end{aligned}
$$

We are now in a position to state the generalised lemma which is one of our results.

Lemma 6.1. The differential operators $A$ and $C$ fit together to form an elliptic complex over $\mathcal{X}$

$$
0 \rightarrow C^{\infty}\left(\overline{\mathcal{X}}, \mathbb{R}^{k-1}\right) \stackrel{C}{\longrightarrow} C^{\infty}\left(\overline{\mathcal{X}}, \mathbb{R}^{k}\right) \stackrel{A^{*}}{\longrightarrow} C^{\infty}(\overline{\mathcal{X}}) \rightarrow 0 .
$$

Proof. The Laplacian $\Delta^{0}$ of (6.2) at step 0 is elliptic, for

$$
\Delta^{0}=C^{*} C=-E_{k-1} \Delta
$$

according to (6.1).

Since $D$ is a square matrix of scalar differential operators with constant coefficients, we deduce that 


$$
-E_{\ell} \Delta=D D^{*}=(A, C)\left(\begin{array}{l}
A^{*} \\
C^{*}
\end{array}\right)=A A^{*}+C C^{*} .
$$

Hence it follows that the Laplacian $\Delta^{1}=A A^{*}+C C^{*}$ of complex (6.2) at step 1 is elliptic.

Finally, the Laplacian $\Delta^{2}$ of (6.2) at step 2 is elliptic, for

$$
\Delta^{2}=A^{*} A=-\Delta
$$

by (6.1).

\section{Conclusion}

In this paper, we proposed a method solving a nonregular boundary value problem for the Cauchy-Riemann operator in $\mathbb{R}^{2}$. Nonregular in the sense, that only the component $u_{1}$ is given on the whole boundary of our domain. We even proposed an exolicit solution to our problem. The next work will be to build an explicit formula for the Laplacian of (6.2) allowing us to construct a fundamental solution of convolution type for the complex (6.2). It is precisely a homotopy formula for the complex (6.2).

\section{Conflicts of Interest}

The authors declare no conflicts of interest regarding the publication of this paper.

\section{References}

[1] Palamodov, V. and Tarkhanov, N. (2009) Nonregular Boundary Problems for Elliptic Systems. Advances in Applied Clifford Algebras, 19, 427-440.

https://doi.org/10.1007/s00006-009-0159-2

[2] Booss-Bavnbek, B. and Wojciechowski, K. (1993) Elliptic Boundary Problems for Dirac Operators. Birkhäuser, Basel. https://doi.org/10.1007/978-1-4612-0337-7

[3] Tarkhanov, N. (1995) Complexes of Differential Operators. Kluwer Academic Publishers, Dordrecht, NL. https://doi.org/10.1007/978-94-011-0327-5

[4] Ly, I. and Tarkhanov, N. (2010) Local Boundary Problem for Dirac Type Operators, Siberian Mathematical Journal, 51, 847-859. https://doi.org/10.1007/s11202-010-0085-5

[5] Tarkhanov, N. (1995) The Cauchy Problem for Solutions of Elliptic Equations. Academie Verlag, Berlin. 